\title{
LES PINERAIES DE CALABRE ET DE SICILE
}

\author{
PAR \\ E.-F. DEBAZAC \\ Directeur de la Station d'Ecologie Forestière \\ C.N.R.F., Nancy
}

Le Pin de Calabre est bien connu des forestiers français. Sa renommée chez nous vient de la réussite de son introduction par Ph.A. de Vilmorin sur le Domaine des Barres au début du Xix ${ }^{\mathbf{e}}$ siècle. En effet, cette essence a été utilisée dans une des premières expériences forestières puisque le but poursuivi par $\mathrm{Ph}$.- $\mathrm{A}$. de VILmoRIN était de comparer les valeurs relatives, comme essences de reboisement, du Pin de Calabre, du Pin laricio de Corse et de différentes provenances de Pin sylvestre. Delamarre [3] donne quelques détails historiques sur cette introduction du Pin de Calabre et rapporte que les graines utilisées furent reçues " directement du pays dans les trois années 1815,1820 et 1821, en une quantité assez considérable d'environ cent livres pesant ou trois millions de graines ». Ces premières plantations, de 1823, furent d'ailleurs complétées vers 1835, par l'introduction du Pin de Crimée [15]. On sait que le Pin de Crimée, comme le Pin de Calabre et le Pin laricio de Corse peuvent être considérés comme différentes formes géographiques d'une même espèce collective, Pinus nigra Arn., à laquelles sont rattachés tous les Pins des groupes « laricio » et " Pin noir ).

Les résultats tout à fait remarquables de ces premiers essais de Pin de Calabre, dont la production en volume, dans l'expérience précitée, s'est effectivement révélée supérieure à celle du Pin de Corse (Pourtet J. et Turpin P. [15], Pardé J. et Turpin $P$. [11]), ont incité à utiliser comme source de graines le peuplement initialement constitué. Ainsi des descendances successives ont été mises en place sur le Domaine des Barres. En outre, des graines récoltées sur ces peuplements ont été largement utilisées dans l'ensemble des reboisements français. Il est même probable que la majeure partie des reboisements effectués avec des plants dits " Pins de Calabre ) sont issus en fait de graines récoltées aux Barres.

Cependant, malgré l'intérêt reconnu de cette essence pour le reboisement, il n'existe dans la littérature forestière française que peu de renseignements sur le Pin de Calabre dans son pays d'origine, en dehors d'un article de Hickel R. et Guinier Ph. [8] sur les forêts de la Sila. 
Aussi, nous parait-il utile de donner nos impressions sur les pineraies de Calabre et de Sicile que nous avons pu visiter au cours d'un récent voyage d'études (1).

\section{Répartition géographique}

L'aire spontanée du Pin est en fait divisée en trois régions distinctes ( $c f$. carte):

- Les superficies les plus importantes sont situées au Nord de la Calabre dans le système montagneux de la Sila dont le centre est à la latitude de Cosenza. Il est possible d'y distinguer trois mas-

\section{CARTE SCHEMATIQUE DES PINERAIES DE CALABRE ET DE SICILE}

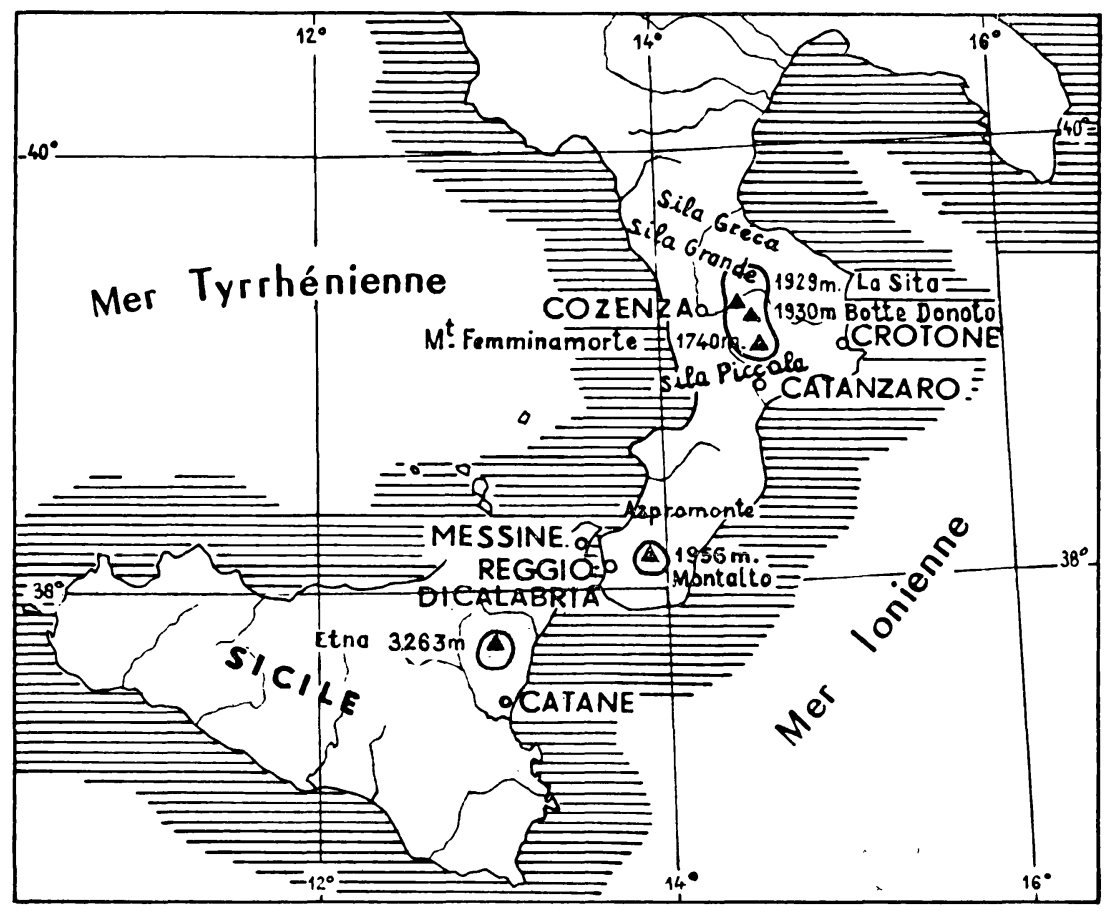

Echelle $1 / 5000000$

(1) Nous tenons à exprimer nos vifs remerciements à M. le Directeur Général des Forêts d'Italie et à tous les personnels des Services régionaux de l'Administration forestière italienne qui nous ont apporté le concours le plus précieux. M. le Professeur Morandini de la Faculté forestière de Florence avait bien voulu établir le programme de cette tournée et nous a donné très aimablement de nombreux renseignements. 
sifs principaux: dans la province de Cosenza, au nord, la Sila Greca; au centre la Sila Grande. Dans la province de Catanzaro, au Sud, la Sila Piccola.

Pour caractériser succinctement le relief de ces montagnes, il faut préciser que le point culminant, le Monte Botte Donato, à 1930 mètres d'altitude, est, à vol d'oiseau, à $35 \mathrm{~km}$ de la Mer Tyrrhénienne et à $40 \mathrm{hm}$ de là Mer Ionienne.

- La seconde région du Pin de Calabre est le massif d'Aspromonte, à l'extrémité de la péninsule dont le point culminant, le Montalto à 1956 mètres d'altitude, est seulement à $17 \mathrm{~km}$ de la mer.

- Enfin, le Pin se rencontre en Sicile sur les versants mêmes de l'Etna dont le point culminant, à $3290 \mathrm{~m}$, est seulement à $20 \mathrm{~km} \mathrm{du}$ littoral.

Pour situer l'importance économique de ces pineraies naturelles, indépendamment des zones de reboisement récent où cette essence a été utilisée largement, il suffit d'ajouter que la superficie est estimée [1] à 45000 ha dans la Sila dont 30000 de peuplements purs, à 6000 ha à Aspromonte dont 5000 de peuplements purs et à 2500 ha environ en Sicile.

L'estimation de ces superficies ne peut être qu'approximative car d'une part le Pin de Calabre vit parfois en mélange avec d'autres essences forestières comme il le sera précisé plus loin et d'autre part certaines propriétés privées partiellement boisées sont mises en valeur également par le pâturage. Ces dernières forêts peuvent prendre parfois l'aspect d'un pré-bois montagnard et ne portent alors qu'un matériel restreint à l'hectare.

Ainsi malgré l'imprécision sur l'étendue exacte de la Pineraie calabraise, il est possible de dire, en la comparant aux peuplements de Pin laricio de Corse, que les forêts de Pin de Calabre ont une superficie supérieure à celles de la Corse évaluée seulement à 22000 hectares [7].

Dans l'ensemble de la Sila, c'est entre 900 et 1500 mètres d'altitude que se trouve la plus grande partie des peuplements de pins.

A Aspromonte, les peuplements sont surtout développés entre 1100 mètres et 1400 mètres, mais il existe des peuplements restreints à plus basse altitude sur le versant Nord à environ 900 mètres d'altitude.

Enfin à l'Etna, dans la forêt de Linguaglossa, la plus importante, la pineraie se développe entre 1150 et 1800 mètres d'altitude.

\section{Conditions écologiques générales}

Il est essentiel de dire tout d'abord que le substratum des pineraies de Calabre est constitué soit par des roches éruptives,, notamment des granites, soit des roches cristallophylliennes, gneiss et micaschistes. 
Toutes ces roches se désagrègent facilement et donnent des sols généràlement profonds. Malgré le relief et le régime pluviométrique de la région, l'activité de l'érosion ne donne pas de pentes abruptes sur les hautes terres si bien que les sommets ont des formes de croupes. Il n'en est pas de même d'ailleurs des zones à altitude moins élevée notamment sur le versant ionien de ces montagnes calabraises où au contraire les torrents ont une action érosive intense.

En Sicile, le Pin se rencontre sur les roches volcaniques, produites par les épanchements de l'Etna aux diverses époques.

Le climat de l'ensemble de la Calabre mériterait d'être analysé en détail pour mettre en évidence les corrélations qui existent entre végétation et type climatique.

La carte bioclimatique de la zone méditerranéenne publiée par l'U.N.E.S.C.O. - F.A.O. [4] rattache la Calabre et la Sicile aux climats thermoméditerranéens et mésoméditerranéens: à basse altitude, le climat est rapporté au type thermoméditerranéen atténué avec un indice xérothermique (1) compris entre 100 et 125 . Aux altitudes plus élevées, donc dans la zone des pineraies, le climat est du type mésoméditerranéen accentué avec un indice xérothermique compris entre 75 et 100 .

D'autres interprétations climatiques ont été données, mais pour donner un des caractères les plus importants du climat, il suffit de citer les précipitations annuelles des stations suivantes:

A Camigliatello, à $1290 \mathrm{~m}$ d'altitude, au cœur du massif de Pins cle la Sila Grande, les précipitations annuelles sont de $1600 \mathrm{~mm}$ [6].

Dans la Sila Piccola, à Villagia Mancuso, à $1250 \mathrm{~m}$ d'altitude, les précipitations annuelles sont de $1650 \mathrm{~mm}$.

Dans l'Aspromonte, à Gambarie, à proximité de la pineraie de Garibaldi, sur le versant nord, à $1300 \mathrm{~m}$ d'altitude, il tombe 1780 mm par an.

Dans toutes ces stations, la neige est abondante chaque hiver et une couche de un à deux mètres n'y est pas rare.

Les observations pluviométriques effectuées dans la pineraie de Castiglione, à l'Etna, ont donné pour la période 1951-1955 une moyenne de $1175 \mathrm{~mm}$ [12].

Le climat de la zone située au-dessus de $1000 \mathrm{~m}$ d'altitude est donc caractérisé par des précipitations annuelles élevées. Le caractère méditerranéen, avec une sécheresse estivale, est net mais atténué puisque dans les trois premières stations citées, la pluviosité estivale est tout de même de 80 à $100 \mathrm{~mm}$ [6].

En raison du relief, il y a évidemment un gradient climatique très marqué entre le littoral et la zone montagneuse. A Crotone, sur le

(1) Indice tendant à traduire la sécheresse biologique en tenant compte à la fois des précipitations, des brouillards et de l'état hygrométrique de l'air. 




Paysage de la Sila grande

près du lac Arvo à $1300 \mathrm{~m}$ d'altitude.

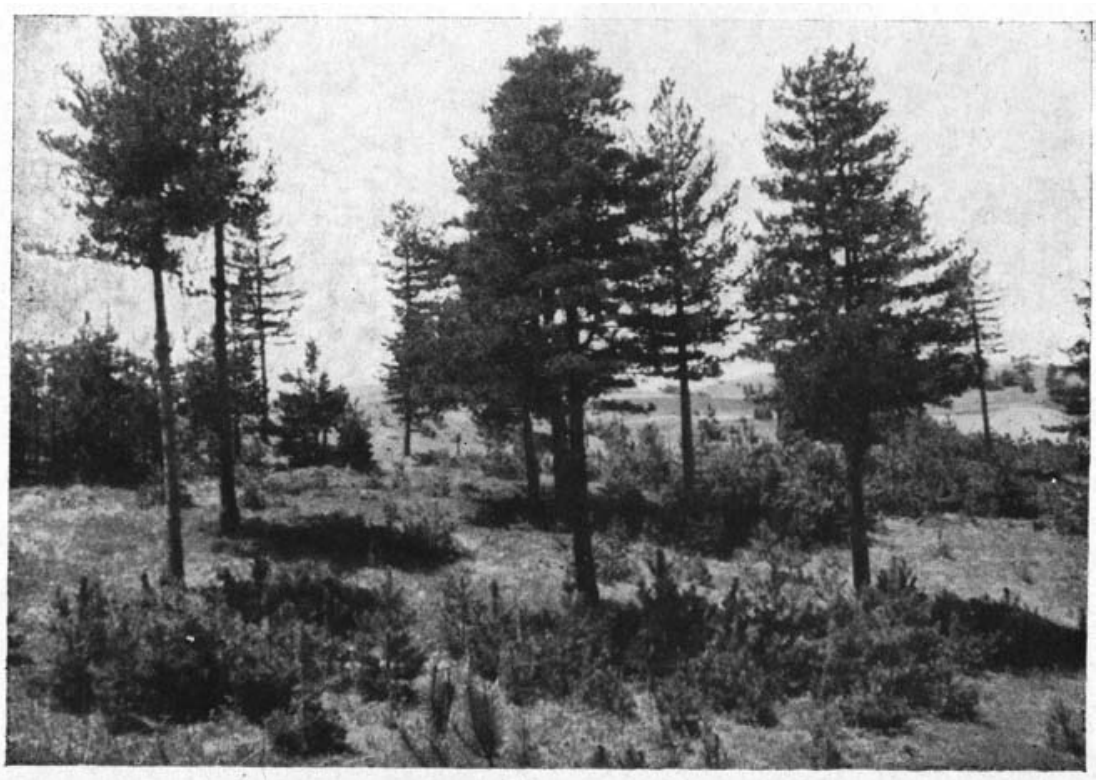

Aspect de pré-bois dans la Sila grande près de Colle d'Ascione dans la Sila grande, à $1300 \mathrm{~m}$ d'altitude. 
littoral ionien, au pied de la Sila, les précipitations annuelles sont seulement de $700 \mathrm{~mm}$, avec un climat méditerranéen du type semiaride; de même, à Reggio Calabria, au pied de l'Aspromonte, les précipitations annuelles sont inférieures à $600 \mathrm{~mm}$. D'ailleurs, la végétation traduit, peut-être mieux que les données climatologiques, ce gradient.

\section{La végétation}

Les étages bioclimatiques en Italie ont été interprétés par de nombreux auteurs.

Ainsi, d'après les zones de végétation distinguées par PavaRI [13], on rencontre en Calabre et en Sicile, successivement à partir du littoral vers l'intérieur, le Lauretum, avec un sous-étage chaud jusqu'à $200 \mathrm{~m}$ d'altitude et un sous-étage froid jusqu'à 600$700 \mathrm{~m}$, puis le Castanetum avec le sous-étage chaud jusqu'à 600 à $900 \mathrm{~m}$ et la sous-zone froide jusqu'à 800-1 $200 \mathrm{~m}$, puis le Fagetum au-dessus de $1200 \mathrm{~m}$. Dans cette classification des étages végétaux, le Pin de Calabre se trouve à la limite du sous-étage froid du Castanetum et à la base du Fagetum.

Pour LÜDI [9], trois régions climatiques se succèdent en altitude: celle caractérisée par le Chène vert jusqu'à environ $200 \mathrm{~m}$ d'altitude, celle du Chêne pubescent jusqu'à $1200 \mathrm{~m}$ dans les Apennins méridionaux, puis celle du Hêtre. Dans cette interprétation, les pineraies se situent encore à la limite des étages du Chêne pubescent et de la hêtraie. Cette distinction des étages altitudinaux d'après les principales essences forestières est très commode. Mais en réalité, le contraste entre le littoral et les sommets est encore plus net puisqu'il y a lieu d'ajouter à la base de l'étage du chêne vert un étage encore plus chaud qui est celui de l'Oleo ceratonion comme l'a indiqué Molinier [10]. Cet étage est caractérisé par la présence dans les formations du type maquis méditerranéen du Lentisque (Pistacia lentiscus L.), du myrte (Myrtus communis L.), du caroubier (Ceratonia siliqua L.), d'une Euphorbe de grande taille (Euphorbia dendroides L.) et d'une graminée puissante (Ampelodesmos fenax Link). Dans le lit des torrents près du littoral se trouvent aussi les stations spontanées de Laurier rose (Nerium oleander L.).

En ce qui concerne les étages de végétation en Calabre, il est intéressant de noter que les plus hauts sommets, le Monte Botte Donato et l'Aspromonte, sont couverts entièrement par la hêtraie. Il n'existe ni étage alpin ni même d'étage pseudo-alpin dû̀ à un effet local des vents. Il n'en est pas de même en Sicile, où, sur l'Etna, la végétation forestière, avec le bouleau, s'arrête vers $2000 \mathrm{~m}$ d'altitude. Au-dessus existe une brousse basse épineuse où domine un arbrisseau endémique, Astragalus siculus. 


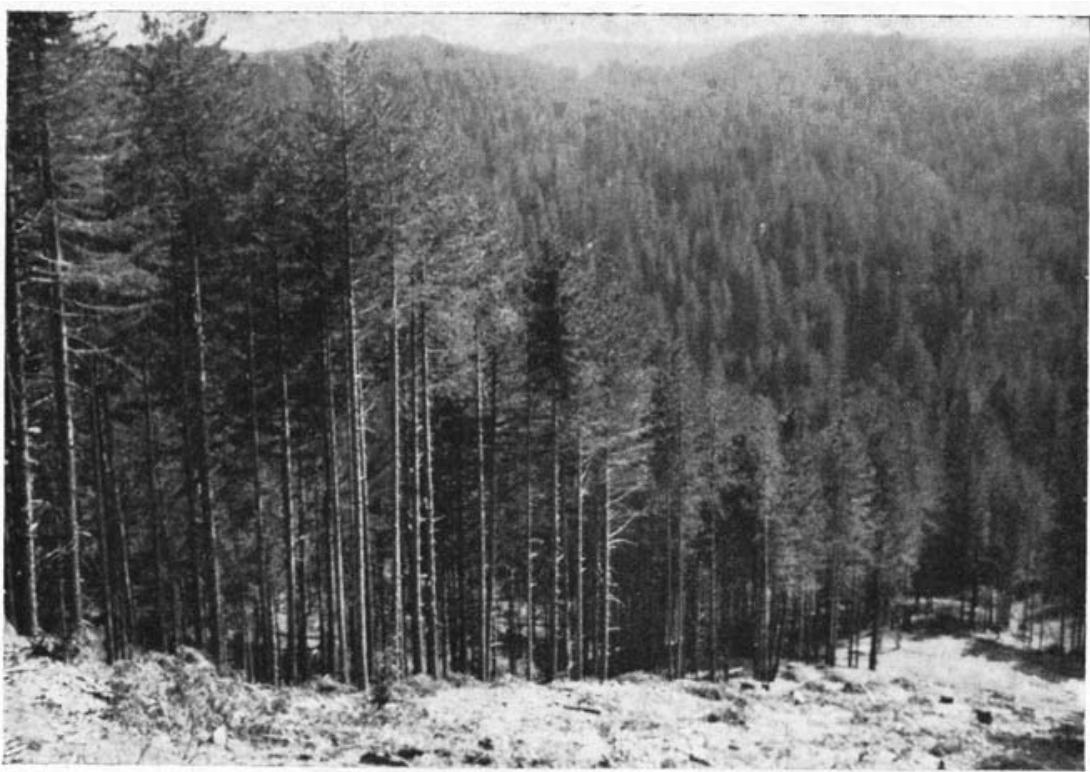

Exploitation par bandes en forêt de Roncino dans la Sila piccola.

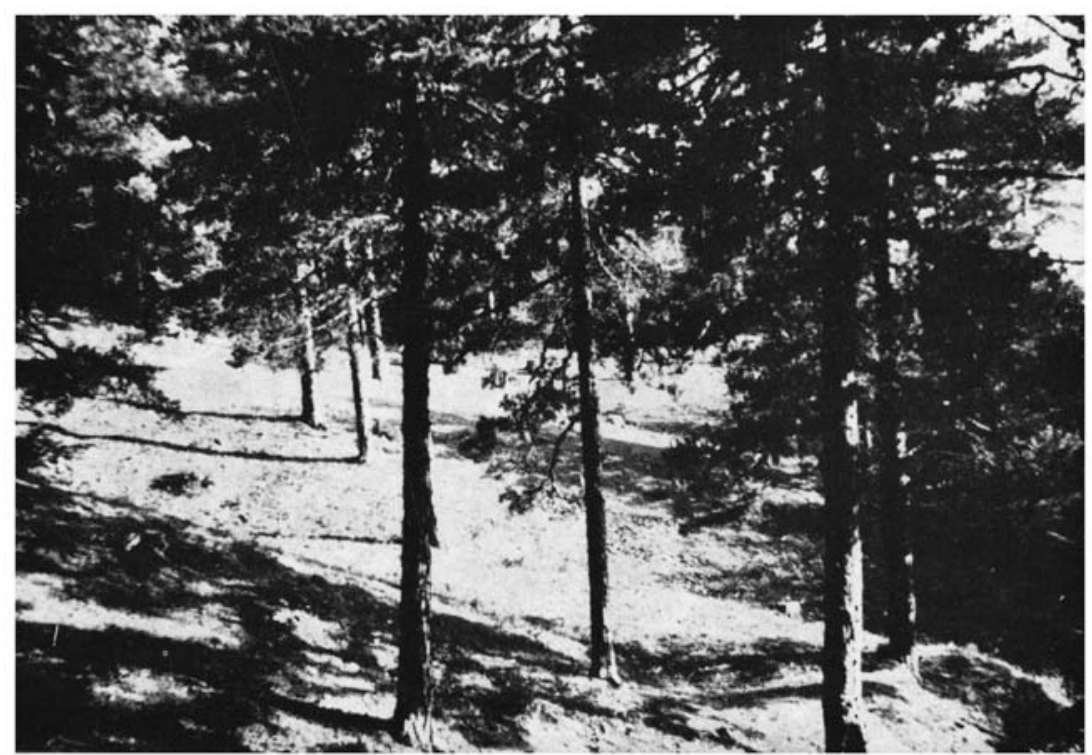

Pineraie sèche sans sous-bois en forêt de San Lorenzo, dans le massif d'Aspromonte. 
Ainsi, en Calabre, la hêtraie, avec quelquefois du sapin en mélange, constitue autant de noyaux de végétation dans l'étage montagnard humide. Au contact de cette hêtraie d'une part et, par ailleurs, au contact de chênaies, soit à chêne pubescent, soit à chêne chevelu (Quercus cerris) ou à Quercus conferta, c'est le domaine de la Pineraie.

L'étude de la flore des pineraies montre qu'il n'existe pas un seul type de groupement végétal de la pineraie mais que les peuplements de Pin se superposent à des groupements qui se rapprochent soit de la hêtraie, soit de la chênaie, soit de pelouses de dégradation sur sols sableux. Quelques exemples pris dans différentes stations permettent d'illustrer l'interprétation de ces pineraies.

Ainsi, dans le massif de l'Aspromonte, sur le versant nord, à 1200 m d'altitude, près de Gambarie, dans la pineraie de Garibaldi, le peuplement de pin, sensiblement équienne et âgé de 70 ans environ, est accompagné par un sous-étage abondant de hêtre. Dans la strate herbacée, à côté de la fougère aigle (Pteridium aquilinum) très abondante, existent des espèces typiques du Mull telles que Brachypodium silvaticum (Huds.) R. et S., Viola silvestris (Link) Rchb., Melica uniflora Retz, Galium rotundifolium L.: il s'agit en réalité d'un lambeau de pineraie, accolé à la hêtraie voisine où existe une flore très caractéristique avec par exemple Lamium galeobdolon Grantz, Asperula odorata L., Oxalis acetosella L., Luzula pedemontana Boiss. et R., et, dans les stations les plus fraîches, Saxifraga rotundifolia $\mathrm{L}$. La pineraie naturelle devait être plus étendue sur le replat de la Piani d'Aspromonte, vers $1100 \mathrm{~m}$ d'altitude, presque entièrement cultivé actuellement.

Dans le même massif d'Aspromonte, mais sur le versant sud, vers $1400 \mathrm{~m}$ d'altitude, existent sur la commune de San Lorenzo, dans le Canton de Cropane, des pineraies bien différentes: le peuplement de pin est accompagné d'un maigre sous-bois composé d'éléments méditerranéens tels que Quercus pubescens, $Q$. ilex, de quelques espèces du maquis de dégradation avec Erica arborea et Cistus salvifolius, mais surtout d'une strate herbacée formant une pelouse pâturée caractéristique des sols siliceux avec notamment Festuca ovina L., Cynosurus elegans D., C. echinatus L., Deschampsia flexuosa (L.) Trin., Brachypodium pinnatum (L.) P.B., Anthoxantum odoratum L., Briza maxima L., Bromus tectorum L., Trifolium campestre Schreb., Hieracium pilosella L., Hypochaeris radicata $\mathrm{L}$.

Par ailleurs, dans les pineraies Ragabo, sur la commune de Linguaglossa, sur le versant nord de l'Etna, il est facile de distinguer deux faciès de végétation: à basse altitude jusque vers $1650 \mathrm{~m}$, la flore qui accompagne le Pin est celle de la forêt de chêne pubescent qui a été décrite par Pirola (A.) et Zappala (Ga) [14]. A altitude plus élevée, il y a passage progressif à la formation de la brousse 
subalpine avec Astragalus alpinus et Juniperus hemisphaerica, la hêtraie étant plus localisée. C'est entre ces deux faciès précités, c'est-à-dire entre 1500 et $1700 \mathrm{~m}$ d'altitude que se trouvent les peuplements de pins les plus denses avec un sous-étage très réduit.

Toutefois, dans la Sila Grande et dans la Sila Piccola, les peuplements purs de pins sont très étendus en raison d'un relief plus favorable, il est alors possible de décrire plusieurs faciès de ces pineraies dépourvues de sous-bois.

Ainsi, dans la Sila Grande, dans la forêt du Tasso, près de Camigliatello, à faible distance de la hêtraie, à $1300 \mathrm{~m}$ d'altitude, les pineraies pures et âgées de 90 ans sont accompagnées d'une très riche flore herbacée avec notamment la fougère aigle. Cette flore n'empêche pas alors la régénération naturelle du Pin qui est très abondante. Ce n'est pas le cas de parcelles de la forêt de la Fossiata à l'est du lac Cecita, entre 1100 et $1600 \mathrm{~m}$ où il est indispensable d'intervenir par un travail du sol exécuté mécaniquement par bande pour obtenir la régénération après un ensemencement artificiel. Le sol est alors recouvert par une végétation herbeuse très puissante où se rencontrent par exemple Luzula forsteri, Brachypodium silvaticum, Orobus variegatus Ten., Sanicula europaea, Anthoxantum odoratum.

D'autre part, dans la Sila Piccola, les plus vastes étendues de pineraies pures se trouvent entre 1200 et $1400 \mathrm{~m}$ d'altitude dans les forêts de Monaco et de Roncino, adossées aux hêtraies-sapinières du sommet du Monte Femminamorta et notamment du Bosco Gariglione. La fougère aigle est très abondante et certaines espèces du sous-bois, s'ajoutant à celles citées ci-dessus pour la Sila Grande, donnent une physionomie plus méditerranéenne. Ainsi, Cytisus sessilifolius est fréquent.

Un des faciès les plus curieux est celui qui semble localisé sur les versants sud, caractérisé par une abondance de Lithospermum calabrum, mêlé à la fougère aigle.

Il est donc certain que le domaine du Pin de Calabre se situe écologiquement entre deux domaines différents: d'une part le domaine du Sapin $\downarrow$ Hêtre, qui occupe d'une façon exclusive les régions les plus montagnardes; d'autre part le domaine méditerranéen de la chênaie pubescente et par place même de la forêt de chêne vert. Le Pin de Calabre occupe ainsi, dans l'ensemble de la végétation forestière, une place homologue à celle du Pin laricio en Corse [2].

Il faut noter toutefois des différences sensibles entre la végétation forestière de la Calabre et celle de la Corse; tout d'abord, en Calabre, existent encore des essences forestières dont l'aire d'extension est le Sud-Est de l'Europe et qui se trouvent là à leurs limites Ouest. C'est le cas de Quercus cerris et $Q$. conferta. 


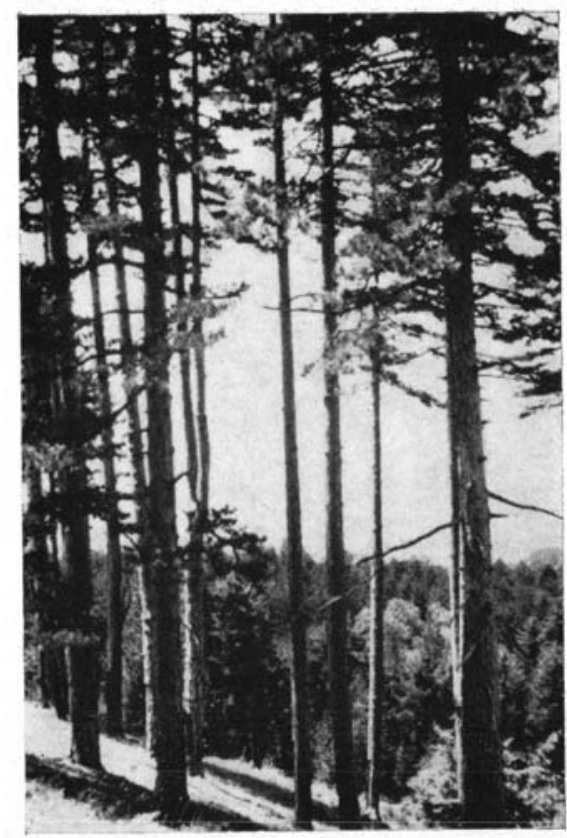

Peuplements âgés en forêt de la Fossiata, dans la Sila grande.

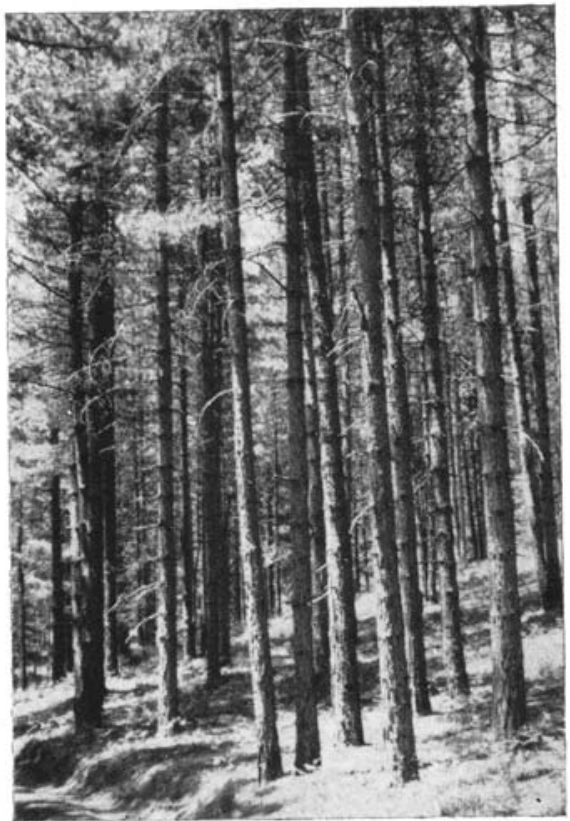

Jeunes pineraies pures de 60 ans près de Villagia Mancuso, dans la Sila piccola.

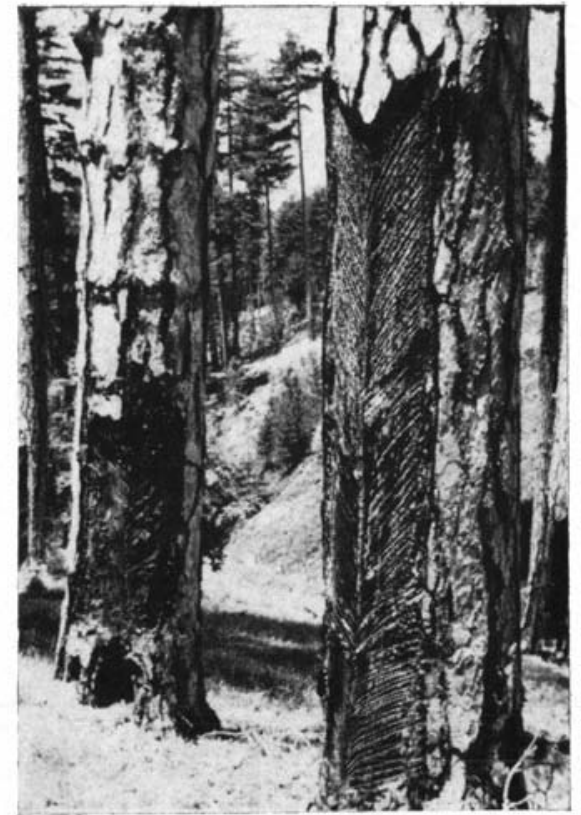

Quelques vieux pins anciennement gemmés en forêt de la Fossiata.

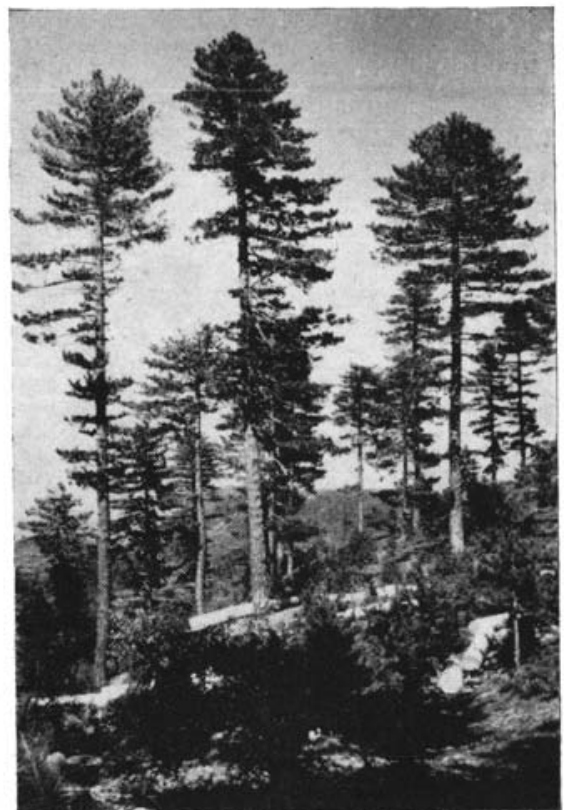

Pineraie en cours d'exploitation en forêt de Ragabo, sur les flancs de l'Etna, à $1700 \mathrm{~m}$ d'altitude. 
D'autre part, l'étage montagnard à Hêtre-Sapin est bien développé au-dessus de $1400 \mathrm{~m}$ d'altitude et occupe tous les sommets alors qu'il n'existe pas d'étage subalpin. Il n'en est pas de même en Corse où la hêtraie et la sapinière ont une place plus réduite et où la variété endémique de l'aune vert (Alnus viridis var. suaveolens) comme la brousse épineuse à Juniperus nana et Berberis aetnensis constituent une formation subalpine très caractéristique des plus hauts sommets, pouvant succéder directement aux stations les plus élevées de la pineraie.

Enfin, le Pin maritime (Pinus pinaster Sol.) ne fait pas partie de la flore forestière de Calabre alors qu'il est aussi important en Corse que le Pin laricio, avec lequel il partage la zone montagnarde des résineux.

\section{Production ligneuse et sylviculture des pineraies}

Le Pin de Calabre atteint des dimensions remarquables puisque des sujets de plus de $45 \mathrm{~m}$ de hauteur totale et de plus de $1,50 \mathrm{~m}$ de diamètre ont été signalés [1]. Ces dimensions sont toutefois exceptionnelles car les chablis touchent fréquemment les sujets âgés (1) qui ne se rencontrent que dans des stations abritées. En fait, l'arbre de 0,45 à $0,50 \mathrm{~m}$ de diamètre, âgé de 80 à 100 ans, est considéré comme ayant atteint les dimensions d'exploitabilité. La productivité moyenne est estimée à $7 \mathrm{~m}^{3}$ par ha et par an dans les bonnes parcelles de la Sila Grande. Les pineraies pures des forêts domaniales sont traitées en futaie simple. Les coupes de régénération sont ouvertes par bandes de $20 \mathrm{~m}$ de large. La régénération est très variable, quelquefois très abondante ou au contraire rare et elle doit être alors complétée artificiellement.

Une partie notable de la production est récoltée en chablis. Le gemmage a été autrefois pratiqué (cf. cliché 6), mais il est actuellement abandonné. Dans les forêts privées, des réalisations de matériel importantes ont été faites depuis la fin de la dernière guerre. En plus, ces forêts sont souvent intensément pâturées mais il faut noter que, malgré cette utilisation pastorale des terrains boisés, des régénérations abondantes s'installent cependant. Le Gouvernement italien poursuit d'ailleurs l'achat des terrains forestiers privés tout en favorisant par ailleurs leur reboisement.

(1) Par exemple, des chablis de $60000 \mathrm{~m}^{3}$ ont été exploités à la suite de coups de vent de décembre 1962 dans la forêt de la Fossiata, dans la Sila Grande.

\section{BIBLIOGRAPHIE}

1. Anzilottr (F.). - 1950. Il pino laricio silano. Monti e Boschi, 3, 1950, pp. 107-116.

2. Debazac (E.-F.). - 1964. Le Pin laricio de Corse dans son aire naturelle.

Revue Forestière Française, 3, 1964, pp. 188-215. 
3. Delamarre. -1831 . Culture des Pins. Paris.

4. F.A.O. - U.N.E.S.C.O. - 1963. Etude écologique de la zone méditerranéenne.

Carte bioclimatique de la zone méditerranéenne.

5. GiacCOBE (A.). - 1937. Ricerche ecologiche e technique sul Pinus laricio Poir. e sul Pinus nigricans Hoess.

Nuovi Annali dell'Agricoltura, XVII, 166 p.

6. Giaccobe (A.). - 1962. Problemi di bioclimatologia mediterranea. Italia forestale e montana, XVII, 4.

7. Guislain (A.). - 1955. La forêt corse. Rivières et Forêts, 3, pp. 4-18.

8. Hickel (R.) et GUINIER ( $\mathrm{Ph}$.). - 1926. Une visite aux pins laricio de la Sila (Calabre).

Bulletin de la Société Dendrologique de France, 60, pp. 81-93.

9. LÜDI (W.). - 1935. Beitrag zur regionalen vegetationgliederung der Appennin halbinsel.

Ergebn. der intern. Pflanzengeogr. Exk - durch Mittelitchen, 1934. Veröff des Geob - Inst. Rübel, pp. 212-239.

10. Molinier (René) et Molinier (Roger). - 1955. Observations sur la végétation littorale de l'Italie occidentale et de la Sicile. Archivio botanico, XXXI, $3^{e}$ série. Vol. XV, fasc. III, 35 p.

11. Pardé (J.) et TURPIN (P.). - 1959. Caractéristiques et production des peuplements de Pins laricio du Domaine des Barres. Revue Forestière Française, no ${ }^{\circ}$, 1959, pp. 376-391.

12. Patrone (G.). - 1964. Piano di Assestamento dei Boschi del Comune di Linguaglossa. Per il Decennio 1963-1972.

86 p. Bruno Coppini et Cie, Firenze, 1964.

13. Pavari (A.). - 1916. Studio preliminare sulla coltura di specie forestali esotiche in Italia. I. parte generale.

Annali del R. Ist. Sup. Forestale Naz. Vol. I.

14. Pirola (A.) et Zappala (Ga). - 1960. La foresta a Pinus laricio Poir. di Linguaglossa (Sicilia).

Bolletino dell'Istituto Botanico della universita di Catania. Série II. Vol. III, 1959, pp. 1-34.

15. Pourtet (J.), Rol (R.) et Turpin (P.). - 1954. Catalogue des espèces cultivées à l'Arboretum des Barres.

Annales de l'Ecole Nationale des Eaux et Forêts, IX, 1, 254 p. 